\title{
Prevalence of Malaria Parasites Amongst Pregnant Women in Calabar Cross River State Nigeria
}

\author{
Bernice Udoh" \\ University of Calabar, Calabar, Nigeria \\ Aniekan Peter \\ University of Uyo, Uyo, Nigeria \\ Sony-Shakins Umotong \\ University of Uyo Teaching Hospital, Uyo, Nigeria \\ A. Alaribe \\ University of Calabar, Calabar, Nigeria
}

\begin{abstract}
Malaria infection during pregnancy is a major public health problem- especially in tropical and sub-tropical regions; with substantial risks for the mother, her foetus and the new-born, Pregnant women are particularly susceptible to malaria, and in low transmission settings they have a greater risk of severe Plasmodium falciparum malaria. This study aimed at investigating the rate of parasitaemia amongst pregnant and none pregnant women was conducted among people attending clinics at the University of Calabar Teaching Hospital, Mambo hospital, Nosam medical laboratory services in Calabar Cross River state. They fall within the age group 18 to 60 years. They were pregnant women who came for Antenatal clinic, controls were sampled from patients who came to request for medical examination and blood donors. Thick and thin blood smears were made from finger prick samples of 400 candidates attending Antenatal clinics 200 of them were from established pregnant women and the other 200 were from nonpregnant women to serve as control. The blood films were dried and fixed in absolute methanol dried and stained with $2 \%$ Giemsa stain for $30 \mathrm{~min}$. it was then rinse in clean water and allowed to dry in a draining rack. Dried slides were viewed using x100 oil immersion objective. Result revealed a parasitic rate of $132(55.9 \%)$ among pregnant women and104 (44.1\%) parasitaemia among none pregnant women. The difference between pregnant women and none pegnant women were significantly different at $\mathrm{p}<0.05$. Similarly, the mean parasite density of the pregnant women was higher 28.9 against 14.2 of control none pregnant women. In conclusion there was a significant density of malaria parasitaemia amongst pregnant women in this study.
\end{abstract}

Keywords: Malaria; Parasitaemia; Pregnancy; Blood films.

\section{(c) (1) CC BY: Creative Commons Attribution License 4.0}

\section{Introduction}

Malaria is a major public health problems in Nigeria and indeed many parts of sub-saharan Africa. Both infections kill millions of people each year with disproportionate heavy burden on Africa, India, Southeast Asia and South America [1,2].

Sub-Saharan Africa accounts for $90 \%$ of the estimated 212 million cases of malaria that causes over 429000 deaths annually [3]. Malaria is a major cause of maternal anemia, which in turn is a risk factor for maternal mortality resulting in about $35 \%$ of preventable cases of low birth weight [4]. Given this magnitude of disease burden, estimating the economic burden of malaria is necessary to provide a basis or platform for advocacy with Ministries of Finance and donors for increased investments in addressing public health problems such as malaria [5]. Data on the economic burden of malaria provide essential information on the magnitude of the problem. They complement disease burden data and can be used to show who bears the economic burden of malaria. Studies have shown that although there are equal exposure and incidence of malaria across the socio-economic groups [6], In Nigeria, malaria has been shown to account for over $40 \%$ of the total monthly curative healthcare costs incurred by households compared to a combination of other illnesses; the cost of treating malaria and other illnesses depleted $7.03 \%$ of the monthly average household income, and treatment of malaria cases alone contributed $2.91 \%$ of these costs [7].

\section{Methods}

\subsection{Study Site}

The study was carried out in Calabar which is the capital of ross river state lies within the tropical rain forest belt which favours the breeding of vectors of parasitic diseases. It has a latitude of $45 \mathrm{~S}$ and $58 \mathrm{~N}$. and the temperature ranges between 25.9 to $34.7{ }^{\circ} \mathrm{c}$. The city has an annual rainfall of about $265 \mathrm{~mm}$ and relative humidity 
range of 77-91. Calabar is highly populated with Efiks, Ibibios Anangs, Ibos and some minor tribes from Northern Cross River state.

\subsection{Study Setting}

This study was conducted among people attending clinics at the University of Calabar Teaching Hospital, Mambo hospital, Nosam medical laboratory services and other private laboratories. They fall within the age group 18 to 60 years. They were pregnant women who came for Antenatal clinic, controls were sampled from patients who came to request for medical examination and blood donors.

\subsection{Collection and Processing of Samples}

Thick and thin blood smears were made from finger prick samples of 400 candidates attending Antenatal clinics 200 of them were from established pregnant women and the other 200 were from non-pregnant women to serve as control. The blood films were air dried and fixed in absolute methanol dried and stained with $2 \%$ Giemsa stain for 30 min. it was then rinse in clean water and allowed to dry in a draining rack. Dried slides were viewed using x100 oil immersion objective.

\subsection{Quantitative Parasite Count}

The thick film was used for the quantitative parasite count. The number of parasite present in the slide were enumerated as described by Shute 1998 as follows

Number of parasites per ul of blood $=$

Number of parasite seen X 8000 1000 leucocytes

\section{Results}

Prevalence of MP among pregnant women samples showed a parasitic rate of 132 (66\%) and104 (52\%) parasitaemia among none pregnant women. The difference between pregnant women and HIV samples were significantly different at $\mathrm{p}<0.05$. Similarly, the mean parasite density of the pregnant women was higher 28.9 against 14.2 of control none pregnant women.

Table-1. Prevalence of Malaria Parasite Among Pregnant Women and Non-pregnant Women

\begin{tabular}{l|l|l}
\hline Age Limit & $\begin{array}{l}\text { Pregnant-Women With Parasite } \\
\text { Load \% }\end{array}$ & $\begin{array}{l}\text { Non-Pregnant Women With } \\
\text { Parasite Load \% }\end{array}$ \\
\hline $18-27$ & $35(26.5)$ & $43(41.3)$ \\
\hline $28-37$ & $82(62.1)$ & $54(51.9)$ \\
\hline $38-47$ & $15(11.4)$ & $7(0.06)$ \\
\hline parasitaemia & $132(66)$ & $104(52)$ \\
\hline No parasitaemia & $68(34)$ & $96(46)$ \\
\hline Malaria parasite density & 28.9 & 14.2 \\
\hline $\begin{array}{l}\text { Comparing with Total } \\
\text { parasitaemia }\end{array}$ & $132(55.9)$ & $104(44.1)$ \\
\hline
\end{tabular}

\section{Discussion}

Malaria in pregnancy is associated with maternal mortality and anaemia, low birth weight (LBW), stillbirth and severe anaemia of the new born. In high transmission settings mothers are usually asymptomatic but in primigravidae, birthweight is reduced [8], In malaria endemic regions, individuals are constantly exposed to malaria parasites through bites of the mosquitoes. This frequent exposure leads to the development of an effective antidisease immunity, which prevents life-threatening parasite burdens and suppresses the pro-inflammatory responses that cause illness [9]. Malaria infection during pregnancy is a major public health problem- especially in tropical and sub-tropical regions; with substantial risks for the mother, her foetus and the new-born [2, 10]. Most cases of malaria in pregnancy in areas of stable malaria transmission are asymptomatic [11]. Depending on the endemicity of malaria in an area, it can be expected that $1-50 \%$ of pregnant women may carry malaria parasitaemia, especially in the placenta, without noticing it Steketee, et al. [12] [2]. This is attributed to anti-disease immunity acquired during previous exposure that protects against clinical malaria [13]. Pregnant women are three times more likely to suffer from severe diseases as a result of malarial infection compared with their non-pregnant counterparts, and have a mortality rate that approaches 50\% [10]. In our study malaria parasitaemia was higher in the young age group 18-27 and 28-37 than the adult age group. 38-47. This may be because of low socioeconomic status of this age group. They are more venerable to malnutrition and sleeping in areas that makes them venerable to mosquito bites.

Specific population at risk of malaria infection include: young children, pregnant people: with HIV/AIDS, international travellers from non -endemic areas (because they lack immunity). And immigrants from endemic areas and their children living in non-endemic areas and returning to their home countries [14]. In our study the pregnant women were more venerable than non-pregnant women. The World Health Organization (WHO) now recommends that all women in the second or third trimester of pregnancy who have uncomplicated $P$. falciparum malaria should be treated with artemisinin-based combination therapy. 
In conclusion there was a significant density of malaria parasitaemia amongst pregnant women in this study.

\section{References}

[1] Asante, K. P., Zandoh, C., Dery, D. B., Brown, C., Adjei, G., Antwi-dadzie, Y., Adjuik, M., Tchum, K., Dosoo, D., et al., 2011. "Malaria epidemiology in the Ahafo area of Ghana." Malaria Journal, vol. 10, p. 211.

[2] Sani, A. F., Mohammed, D., Abubakar, B., Sule, A. A., Asiya, U. I., and Shehu, U. N., 2015. "Prevalence and risk factors associated with malaria infection among pregnant women in a semi-urban community of north-western Nigeria Infectious Diseases of Poverty." vol. 4, p. 24.

[3] Organization, W. H., 2016. World malaria report 2015: World Health Organization.

[4] Owusu-Agyei, S., Asante, K. P., Adjuik, M., Adjei, G., Awini, E., Adams, M., Newton, S., Dosoo, D., Dery, D., et al., 2009. "Epidemiology of malaria in the forest-savanna transitional zone of Ghana." Malaria Journal, vol. 8, p. 220.

[5] Okorosobo, T., Okorosobo, F., Mwabu, G., Orem, J. N., and Kirigia, J. M., 2011. "Economic burden of malaria in six countries of Africa." Eur J Bus Manage, vol. 3, pp. 42-62.

[6] Uguru, N. P., Onwujekwe, O. E., Uzochukwu, B. S., Igiliegbe, G. C., and Eze, S. B., 2009. "Inequities in incidence, morbidity and expenditures on prevention and treatment of malaria in southeast Nigeria." BMC international health and human rights, vol. 9, p. 21.

[7] Onwujekwe, O., Chima, R., and Okonkwo, P., 2000. "Economic burden of malaria illness versus that of a combination of all other illnesses: A study in five malaria holo-endemic communities." Health Policy, vol. 54, pp. 143-159.

[8] Brabin, B., Maxwell, S., Chimsuku, L., Verhoeff, F., van der Kaay, H. J., Broadhead, R., Kazembe, P., and Thomas, A., 1993. "A study of the consequences of malarial infection in pregnant women and their infants." Parassitologia, vol. 35, pp. 9-11.

[9] Riley, E. M., Hviid, L., Theander, T. G. M., and Kierszenbaum, F., 1994. Parasitic infections and the immune system. New York: Academic Press.

[10] World Health Organization, 2016. "World malaria report 2015, World health organization."

[11] Mockenhaupt, F. P., Ulmen, U., von Gaertner, C., Bedu-Addo, G., and Bienzle, U., 2002. "Diagnosis of placental malaria." J. Clin. Microbiol., vol. 40, pp. 306-308.

[12] Steketee, R. W., Nahlen, B. L., Parise, M. E., and Menendez, C., 2001. "The burden of malaria in pregnancy in malaria-endemic areas." Am. J. Trop. Med. Hyg., vol. 64, pp. 28-35.

[13] Staalsoe, T., Shulman, C. E., Buhner, J. N., Kawuondo, K., Marsh, K., and Hviid, L., 2004. "Variant surface antigen-specific IgG and protection against clinical consequences of pregnancy-associated Plasmodium falciparum malaria." Lancet, vol. 363, pp. 283-289.

[14] Ruiz Lopez, d. P. G., Hernán, G. C., Moreno, C. L., Fernández, E. V., Muñoz, M. M. F., Delgado, M. A., Polo, P. M. J., and Andrés, G. I., 2014. 